\title{
Dosimetric comparison of intensity-modulated radiotherapy (IMRT) and volumetric modulated arc therapy (VMAT) in total scalp irradiation: a single institutional experience
}

\author{
Christian Ostheimer, MD, Patrick Hübsch, MS, Martin Janich, PhD, Reinhard Gerlach, PhD, \\ Dirk Vordermark, MD, PhD \\ Department of Radiation Oncology, Faculty of Medicine, Martin Luther University Halle-Wittenberg, Halle, Germany
}

Purpose: Total scalp irradiation (TSI) is a rare but challenging indication. We previously reported that non-coplanar intensitymodulated radiotherapy (IMRT) was superior to coplanar IMRT in organ-at-risk (OAR) protection and target dose distribution. This consecutive treatment planning study compared IMRT with volumetric-modulated arc therapy (VMAT).

Materials and Methods: A retrospective treatment plan databank search was performed and 5 patient cases were randomly selected. Cranial imaging was restored from the initial planning computed tomography (CT) and target volumes and OAR were redelineated. For each patients, three treatment plans were calculated (coplanar/non-coplanar IMRT, VMAT; prescribed dose 50 Gy, single dose $2 \mathrm{~Gy}$ ). Conformity, homogeneity and dose volume histograms were used for plan.

Results: VMAT featured the lowest monitor units and the sharpest dose gradient (1.6 Gy/mm). Planning target volume (PTV) coverage and homogeneity was better in VMAT (coverage, 0.95; homogeneity index [HI], 0.118) compared to IMRT (coverage, 0.94; $\mathrm{HI}, 0.119$ ) but coplanar IMRT produced the most conformal plans (conformity index [CI], 0.43). Minimum PTV dose range was 66.8\% $-88.4 \%$ in coplanar, $77.5 \%-88.2 \%$ in non-coplanar IMRT and 82.8\%-90.3\% in VMAT. Mean dose to the brain, brain stem, optic system (maximum dose) and lenses were 18.6, 13.2, 9.1, and 5.2 Gy for VMAT, 21.9, 13.4, 14.5, and 6.3 Gy for non-coplanar and 22.8, 16.5, 11.5, and 5.9 Gy for coplanar IMRT. Maximum optic chiasm dose was 7.7, 8.4, and 11.1 Gy (non-coplanar IMRT, VMAT, and coplanar IMRT).

Conclusion: Target coverage, homogeneity and OAR protection, was slightly superior in VMAT plans which also produced the sharpest dose gradient towards healthy tissue.

Keywords: Total scalp irradiation, Intensity-modulated radiotherapy, Volumetric-modulated arc therapy, Angiosarcoma, Mycosis fungoides, Lymphoma of the scalp, Dosimetry

\section{Introduction}

Extensive superficial malignancies of the scalp constitute a rare but challenging clinical presentation in oncology. Most common malignancies treated with total scalp irradiation (TSI) include angiosarcoma, cutaneous lymphoma and squamous cell carcinoma [1]. However, radiotherapy of the scalp can be technically demanding due to the unique clinical presentation

Received 31 August 2016, Revised 22 September 2016, Accepted 14 October 2016.

Correspondence: Christian Ostheimer, MD, Department of Radiation Oncology, Faculty of Medicine, Martin Luther University Halle-Wittenberg, Ernst-Grube-Strasse 40, 06120 Halle, Germany. Tel: +49-345-557-3432, Fax: +49-345-557-4333, E-mail: Christian.Ostheimer@uk-halle.de

(c) This is an Open Access article distributed under the terms of the Creative Commons Attribution Non-Commercial License (http://creativecommons.org/ licenses/by-nc/4.0/) which permits unrestricted non-commercial use, distribution, and reproduction in any medium, provided the original work is properly cited.

www.e-roj.org 
of the tumor on the one side (multifocal spread, irregular borders) and the complex geometry of the scalp on the other side (convex surface, close proximity of visual organs and face).

In addition to the anatomical challenges, intense treatment planning efforts, complex and staff-intensive treatment and patient setup (immobilisation) further complicate this clinical situation. Radiotherapeutic approaches to treat the entire scalp range from electron-based over photon-based to combined techniques including intensity-modulated radiotherapy (IMRT), volumetric-modulated arc therapy (VMAT), tomotherapy and high dose rate (HDR) brachytherapy [2-5].

However, sufficient data to determine the optimal and most effective radiation regimen is still lacking and frequently, both the individual and specific clinical tumor presentation as well as the technical conditions at the institution limit the scope of possible techniques to be chosen.

Achieving an adequate dose coverage in the target volume, that is the dermis and epidermis, while sparing brain parenchyma was historically attempted by matched, shifted or overlapping electron fields [6-8]. However, these techniques not only suffered from significant dose inhomogeneity but also from electron scatter complicating dosimetry [9]. These obstacles could be partially resolved by using photon beam IMRT techniques or photon-electron combination approaches which resulted in improved target dose coverage, dose homogeneity and minimized difficulties with doses at field junctions [4,9-11].

We previously reported our institution's experience and comparatively evaluated coplanar and non-coplanar photon IMRT in the TSI of scalp malignancies under a dosimetric perspective and found that non-coplanar IMRT techniques were superior in dose homogeneity, target volume coverage and sparing of the optical structures [12].

Novel motion-based radiation techniques such as nonstatic photon IMRT and VMAT in particular, promise superior dose distribution and conformity $[13,14]$. Recently, first evidence showed encouraging clinical response in a patient with extensive leukaemia cutis of the head and scalp treated by VMAT [15].

However, a robust analysis comparing static-field IMRT with VMAT has not been undertaken. This retrospective treatment planning study now evaluates the dosimetric merits of IMRT against VMAT and comparatively assesses plan conformity index $(\mathrm{Cl})$, homogeneity index $(\mathrm{HI})$, dose-volume relation and organ-at-risk (OAR) protection in radiotherapy of the whole scalp.

\section{Materials and Methods}

1. Radiation treatment plan calculation and characteristics A patient databank search was performed at the Department of Radiation Oncology of the Martin Luther University HalleWittenberg and 5 patients who previously received whole brain radiotherapy (WBRT) for cerebral metastasis (without prior cranial surgery) were randomly selected. All clinical and imaging information was analysed anonymously. This specific patient group (i.e., with intracerebral metastasis but without malignant scalp lesions) was chosen to ensure homogeneous and identical planning conditions with an anatomically intact and unaltered scalp surface to best evaluate the dosimetric differences of the radiation techniques. Generally, the previous WBRT was based on computed tomography (CT)-images obtained in treatment position (GE LightSpeed RT; without contrast) with the patients' head immobilized in an individual thermoplastic mask without use of a bolus. It was carried out in a three-dimensional (3D) conformal radiation technique.

Treatment plans were restored and the entire prior plan content including previously delineated target volumes and
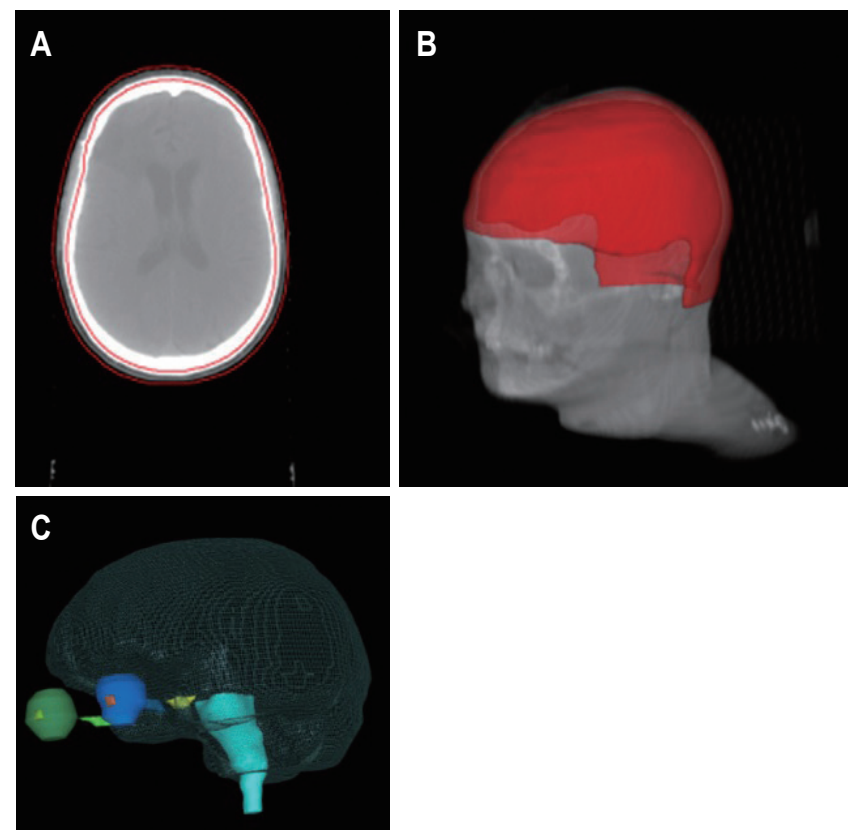

Fig. 1. Three-dimensional reconstruction of planning target volume (PTV) and organs at risk (OAR). (A) Transversal planning computed tomography (CT) with PTV (red, whole scalp). (B) Transversal CT with PTV (red) of patient \#3. (C) OAR of patient \#3 (eyeballs, dark green and blue; brainstem, light blue; optic chiasm, yellow; optic nerves, light green and blue; lenses, orange and light green; brain, grid). 
A
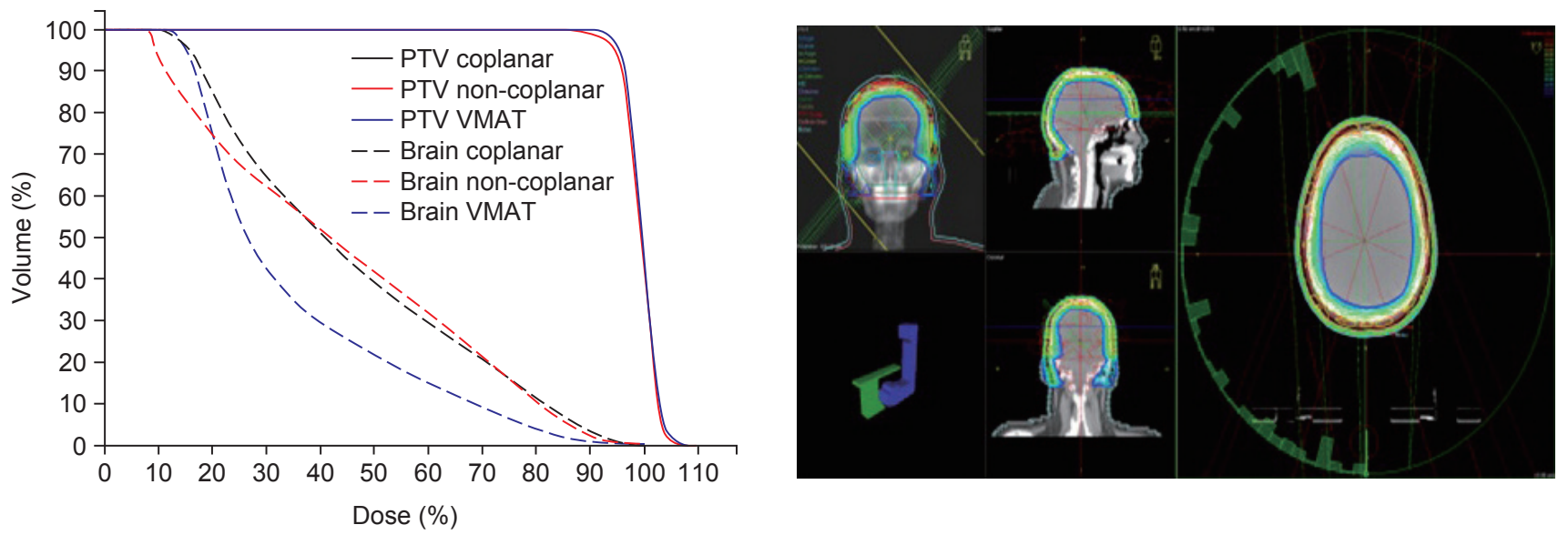

B
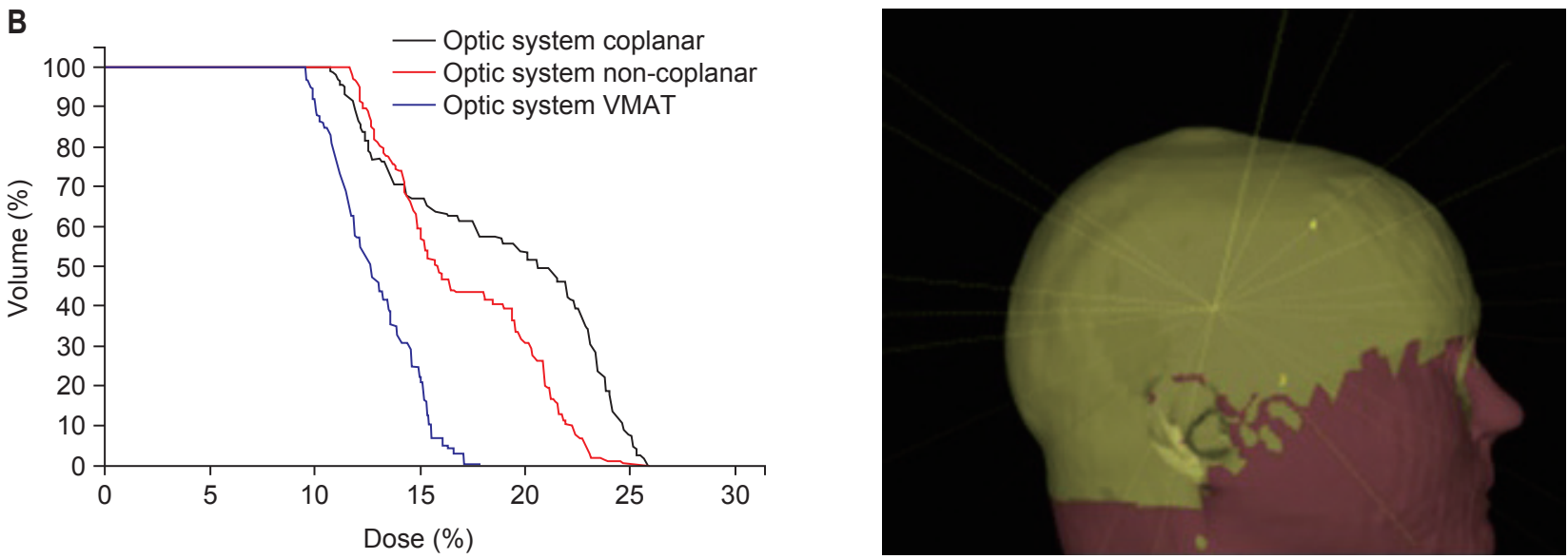

Fig. 2. Three-dimensional (3D) reconstruction of the volumetric modulated arc therapy (VMAT) and non-coplanar intensity-modulated radiotherapy (IMRT) treatment plan including comparative dose-volume histogram (DVH) of patient \#3. (A) Comparative DVH with planning target volume (PTV) dose (continuous line) in coplanar (black) vs. non-coplanar (red) vs. VMAT (blue) plans and brain dose (dashed line) in coplanar (black), non-coplanar (red), and VMAT (blue) plans. (B) Comparative DVH for optic system dose (optic nerves, lenses, eye balls, optic chiasm) in coplanar (black) vs. non-coplanar (red) vs. VMAT plans (blue). (C) PTV reconstruction with dose distribution, arc 1 of 4. (D) 3D reconstruction of scalp surface dose coverage in non-coplanar IMRT with radiation fields 1-11 (yellow lines).

OAR contours were deleted. For each patient, planning target volume (PTV) (i.e., from skin surface to skull cap), OAR (brain, optic chiasm, left and right optic nerve, left and right lens, brainstem) were re-delineated by an experienced radiation oncologist using the Oncentra MasterPlan external beam planning software (Nucletron, Columbia, MD, USA) (Fig. 1). For each patient, three treatment plans were generated using the collapsed cone algorithm: a coplanar IMRT plan, a noncoplanar IMRT plan and a VMAT plan, based on the same PTV of each individual patient, yielding a total of 15 radiation treatment plans for comparison. For IMRT plans, 20 iteration steps per cycle were applied for creating the fluency and 80 iteration steps for optimization and consecutive segmentation of the multi-leaf-collimator. Planning time for IMRT plans ranged from 2 to 4 hours per plan which were calculated as an 11-field step-and-shoot technique. Non-coplanar IMRT used 6 couch angles $\neq 0^{\circ}\left(45^{\circ}, 315^{\circ}, 90^{\circ}\right)$ and VMAT plans were based on 4 arcs each at $180^{\circ}\left(180^{\circ}-340^{\circ}\right.$ clockwise, $20^{\circ}-180^{\circ}$ clockwise, $180^{\circ}-20^{\circ}$ counter clockwise, $340^{\circ}-180^{\circ}$ counter clockwise), with arcs covering the same angle range being a dual arc (Fig. 2). A 5-mm virtual bolus (without air gap between bolus and scalp) was created and used for all treatment plans to ensure sufficient surface dose. Plan calculation time for VMAT ranged from 6 to 8 hours per plan.

Dose prescription for all plans was 50 Gy to the PTV (whole scalp) at fractions of $2 \mathrm{~Gy}$ (6 MV). Radiation doses are reported as maximum dose $\left(D_{\text {max }}\right), D_{2 \%}$, minimum dose $\left(D_{\min }\right)$ and $D_{98 \%}$ and dose gradient across the target volume is reported in Gy 
per mm. Doses at OARs are reported as mean dose in Gy (brain and lenses) or maximum dose in Gy (optical nerves, brain stem, optic chiasm), respectively. For the brain dose constraint, a maximum average dose was used and dose constraints for the PTV were $D_{\text {min }}\left(D_{95 \%}\right) 47 G y, D_{\text {min }}\left(D_{98 \%}\right) 48.5 \mathrm{~Gy}, D_{\text {mean }} 50 \mathrm{~Gy}$, and $D_{\max } 54 \mathrm{~Gy}$. OAR doses were lowered as much as reasonable achievable, that is until a significant deterioration of the plan homogeneity and PTV coverage occurred. Monitor unites (MUs) of each treatment was also recorded to evaluate the execution efficiency.

\section{Dose conformity}

In the literature, different conformity indexes were published [16]. In this study, the conformity index (CI) as defined by Paddick [17] was used:

$$
C l=\frac{T V(P I V)^{2}}{T V \times P I V}
$$

where TV(PIV) refers to the target volume covered by the prescription isodose volume, and TV is the target volume. PIV describes the prescription isodose volume, referring to the $95 \%$ or $98 \%$ isodose. The homogeneity index (HI) was defined according to the ICRU report 83 [18-20] as

$$
H I=\frac{D_{2}-D_{98}}{D_{50}}
$$

with $D_{50}$ being the median absorbed dose and $D_{2}$ and $D_{98}$ representing the doses received by $2 \%$ and $98 \%$ of the PTV. PTV coverage was the ratio of the PTV receiving 95\% of the prescribed dose $\left(\mathrm{V}_{95 \%}\right)$ and the corresponding PTV:

$$
P T \text { coverage }=\frac{T \text { (M5\%) }}{T V}
$$

\section{Results}

\section{Dosimetric analysis IMRT and VMAT treatment plans}

Dosimetric comparison of the coplanar and non-coplanar IMRT and VMAT plans including PTV coverage, homogeneity, conformity, minimum and maximum doses are presented in Table 1 for each individual patient case. Mean MUs for all VMAT plans were 950.8 followed by non-coplanar IMRT plans (962.8) and coplanar IMRT (974.2). Treatment plan delivery was 10-12 minutes for IMRT and 2-3 minutes for VMAT plans on average.
Mean dose gradient was 1.6, 1.1, and $1.3 \mathrm{~Gy} / \mathrm{mm}$ in VMAT, coplanar and non-coplanar IMRT. PTV coverage was 0.94 for coplanar/non-coplanar IMRT and 0.95 for VMAT plans (mean). Mean HI was 0.119 for IMRT and 0.118 for VMAT plans while the mean $\mathrm{Cl}$ was $0.45\left(\mathrm{Cl}_{98}\right)$ in non-coplanar, 0.4 in coplanar IMRT and 0.43 in VMAT plans $\left(\mathrm{Cl}_{95}\right.$ was 0.39 for coplanar IMRT and 0.43 for non-coplanar IMRT and VMAT). Minimum PTV dose (mean) was 43.7 Gy (VMAT), 41.4 Gy (non-coplanar IMRT) and 41.3 Gy (coplanar IMRT); maximum PTV dose (mean) was 54.7 Gy (VMAT, non-coplanar IMRT) and 54.8 Gy (coplanar IMRT). $D_{98}$ was $46.5,46.4$, and 46.6 Gy and $D_{2}$ was 52.2, 52.3, and $52.5 \mathrm{~Gy}$ in coplanar, non-coplanar IMRT and VMAT.

Except for 1 patient (\#2), VMAT produced the sharpest dose gradients of all plans. In 2 patients, PTV coverage was significantly higher in VMAT plans (0.95, patient \#2 and 0.97, patient \#3); while in 2 patients, TV coverage was comparable between treatment plans (patients \#4 and \#5) and in 1 patient (\#1), PTV coverage was superior in the non-coplanar IMRT. HI was comparable between treatment plans (Table 1).

In all patients, non-coplanar IMRT plans were more conformal than coplanar IMRT which applies to 3 out of 5 patients for VMAT.

All treatment plans produced dose hot spots within and outside the target volume, however, they were comparable between treatment plans of each individual patient as were maximum doses which ranged from 107.2-112.6 in coplanar IMRT, from 106.6-112 in non-coplanar IMRT and from 104.8113.3 in VMAT plans which also produced both the lowest (104.8, patient \#5) and the highest maximum dose (113.3, patient \# 1). Minimum PTV doses ranged from 66.8\%-88.4\% in coplanar IMRT plans, from 77.5\%-88.2\% in non-coplanar IMRT plans and from 82.8\%-90.3\% in VMAT plans which produced the highest minimum doses, except for 1 patient (\# 1). Both $D_{98}$ and $\mathrm{D}_{2}$ were similar in IMRT and VMAT plans (Table 1).

Fig. 2 shows a comparative exemplary dose-volumehistogram (DVH) with PTV and mean brain dose in IMRT and VMAT plans of patient \#3, indicating sufficient and comparable PTV dose coverage with all treatment planning techniques and superior brain doses in the VMAT plan. Fig. 3 highlights depth dose characteristics of the VMAT plan from the surface of the scalp of patient \# 1 .

\section{Dosimetric comparison of organ at risk involvement of IMRT and VMAT treatment plans}

Table 2 shows the organ at risk doses for each plan of each individual patients. In all patients, VMAT plans produced considerable lower mean brain doses (range, 13.8 to $21.2 \mathrm{~Gy}$ ) 


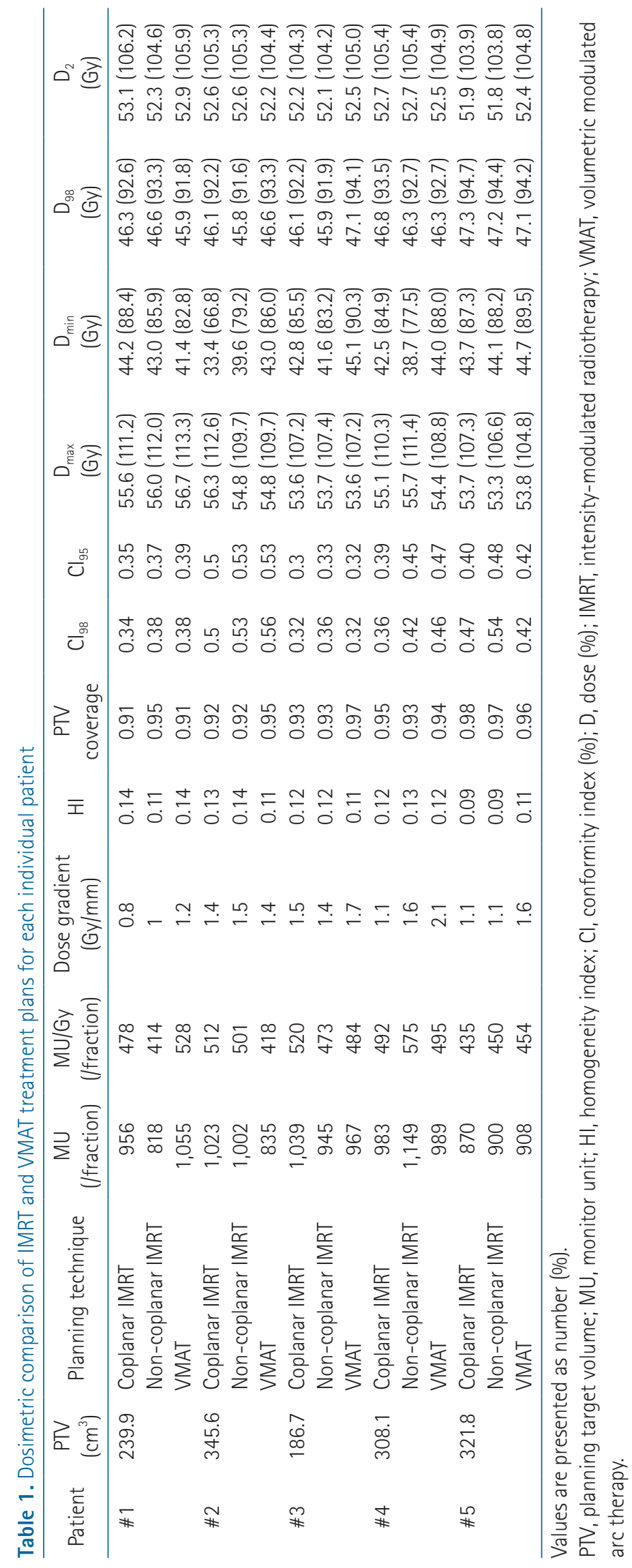


A
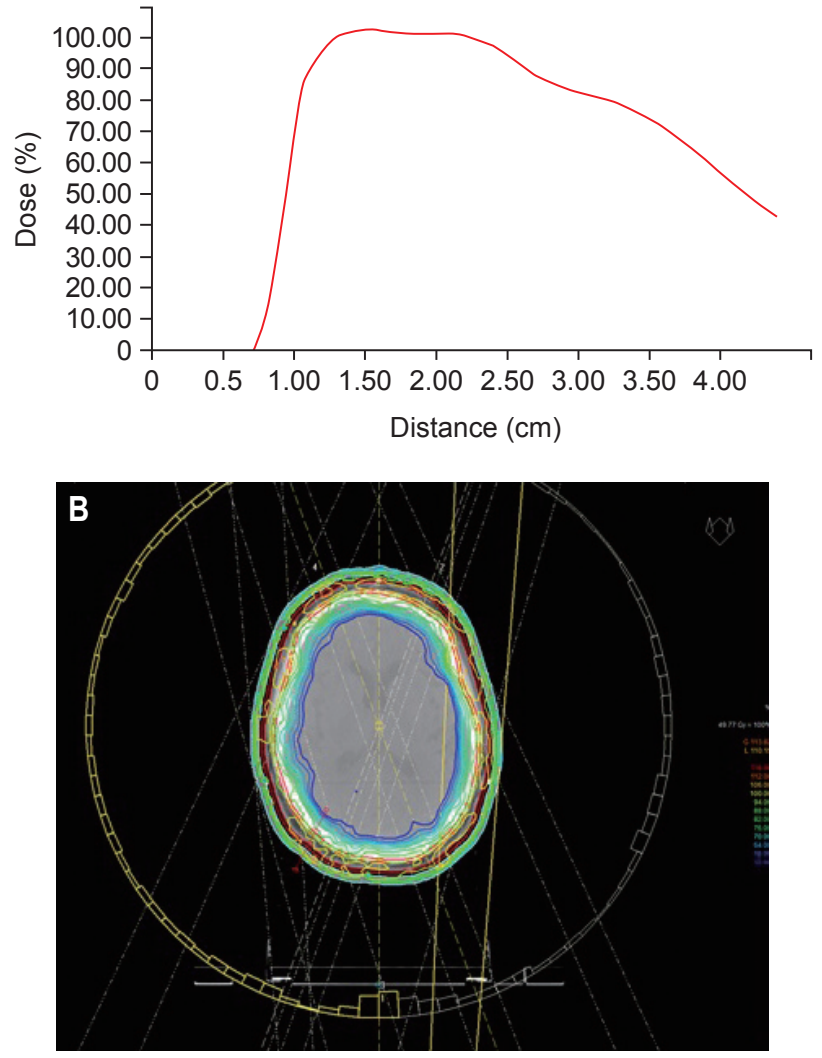

Fig. 3. Volumetric-modulated arc radiotherapy treatment plan (patient \#1). (A) Surface dose profile curve and (B) isodose distribution over scalp planning target volume. compared to non-coplanar (range, 18.7 to $24 \mathrm{~Gy}$ ) and coplanar IMRT plans (range, 19.7 to $25.8 \mathrm{~Gy}$ ). The non-coplanar IMRT technique was slightly superior in mean brain dose.

The mean dose at the brain stem was lowest in VMAT, followed by non-coplanar IMRT and coplanar IMRT in three patients. In 2 patients, brain stem dose was superior in noncoplanar IMRT (Table 2). Maximum dose to the optic chiasm was lower in non-coplanar (range, 3.8 to $12.5 \mathrm{~Gy}$; mean, 7.7 Gy) compared to coplanar (range, 3.8 to 17.6 ; mean, $11.1 \mathrm{~Gy}$ ) IMRT in each of the 5 patient planning cases, while VMAT ranged intermediate (range, 4.7 to $13.3 \mathrm{~Gy}$; mean, $8.4 \mathrm{~Gy}$ ). In 4 patients, VMAT was inferior (range, 4.7 to $13.3 \mathrm{~Gy}$ ) to noncoplanar IMRT (range, 3.8 to $12.5 \mathrm{~Gy}$ ) in regard of mean brain stem dose and in 1 patient, VMAT was superior (5.3 vs. 6.3 Gy) (Table 2).

VMAT plans produced considerably lower doses at the optic nerves (8.2-9.9 Gy) compared to coplanar (8.7-18.5 Gy) and non-coplanar (10.7-21.9 Gy) which had the highest optic nerves dose in all patients. For eye lenses, VMAT was superior in 3 patient cases compared to non-coplanar and coplanar IMRT, however, doses to the lenses overall were comparable (Table 2).

Mean brain dose in all patients was lowest in VMAT (18.6 Gy), followed by non-coplanar (21.9 Gy) and coplanar IMRT (22.8 Gy) which produced similar mean brain doses. Mean brain stem dose was 16.5, 13.4, and $13.2 \mathrm{~Gy}$ in coplanar, non-

Table 2. Dosimetric comparison of organ at risk in IMRT and VMAT treatment plans for each individual patient

\begin{tabular}{|c|c|c|c|c|c|c|c|}
\hline \multirow{2}{*}{ Patient } & \multirow{2}{*}{ Planning technique } & \multicolumn{4}{|c|}{ Mean dose (Gy) } & \multicolumn{2}{|c|}{ Maximum dose (Gy) } \\
\hline & & Brain & Brain stem & Left lens & Right lens & Optic chiasm & Optic nerves $^{\text {a) }}$ \\
\hline \multirow[t]{3}{*}{$\# 1$} & Coplanar IMRT & $23.8(47.6)$ & $22.3(44.5)$ & $4.5(9.0)$ & $6.0(12.0)$ & $17.6(35.2)$ & $18.5(37.0)$ \\
\hline & Non-coplanar IMRT & $23.2(46.5)$ & $18.3(36.6)$ & $5.3(10.6)$ & $6.4(12.8)$ & $12.5(25.0)$ & $21.9(43.8)$ \\
\hline & VMAT & $20.4(40.7)$ & $16.2(32.5)$ & $4.6(9.2)$ & $6.9(13.8)$ & $13.3(26.6)$ & $9.4(18.8)$ \\
\hline \multirow[t]{3}{*}{$\# 2$} & Coplanar IMRT & $22.4(44.8)$ & 16.7 (33.3) & $6.0(12.0)$ & $5.8(11.6)$ & $11.4(22.8)$ & $8.7(17.4)$ \\
\hline & Non-coplanar IMRT & $21.7(43.3)$ & $10.3(20.6)$ & $5.7(11.4)$ & $5.4(10.8)$ & $8.1(16.2)$ & $15.3(30.6)$ \\
\hline & VMAT & $20.5(41.6)$ & $14.1(28.2)$ & $4.8(9.6)$ & $4.6(9.2)$ & $10.1(20.2)$ & $8.5(17.0)$ \\
\hline \multirow[t]{3}{*}{ \#3 } & Coplanar IMRT & $22.6(45.2)$ & $21.2(42.4)$ & 6.5 (13.0) & $6.4(12.8)$ & $13.0(26.0)$ & $12.2(24.4)$ \\
\hline & Non-coplanar IMRT & $21.8(37.4)$ & $18.7(37.4)$ & $6.7(13.4)$ & $6.7(13.4)$ & 7.7 (15.4) & $13.7(27.4)$ \\
\hline & VMAT & $17.2(34.4)$ & $14.9(29.7)$ & $4.8(9.6)$ & $5.0(10.0)$ & $8.7(17.4)$ & $8.8(17.4)$ \\
\hline \multirow[t]{3}{*}{ \#4 } & Coplanar IMRT & 19.7 (39.3) & $6.2(12.4)$ & $5.2(10.4)$ & $5.3(10.6)$ & $3.8(7.6)$ & $10.6(21.2)$ \\
\hline & Non-coplanar IMRT & $18.7(37.4)$ & $4.6(9.2)$ & $6.4(12.8)$ & $5.3(11.6)$ & $3.8(7.6)$ & $10.7(21.4)$ \\
\hline & VMAT & $13.8(27.7)$ & $7.2(14.3)$ & $5.8(11.6)$ & $4.6(7.2)$ & $4.7(9.4)$ & $8.2(16.4)$ \\
\hline \multirow[t]{3}{*}{ \#5 } & Coplanar IMRT & $25.8(51.5)$ & $16.2(32.4)$ & $6.7(13.4)$ & $6.9(13.8)$ & 9.6 (19.2) & $10.7(21.4)$ \\
\hline & Non-coplanar IMRT & $24.0(48.0)$ & $15.2(30.5)$ & $8.1(16.2)$ & $7.3(14.6)$ & $6.3(12.6)$ & $13.2(26.4)$ \\
\hline & VMAT & $21.2(42.4)$ & $13.7(27.4)$ & $5.3(10.6)$ & $5.2(10.4)$ & $5.3(10.8)$ & 9.9 (19.8) \\
\hline
\end{tabular}

Values are presented as number (\%).

IMRT, intensity-modulated radiotherapy; VMAT, volumetric modulated arc therapy.

${ }^{\text {a) }}$ Right and left optic nerve. 
coplanar IMRT and VMAT. Mean dose at optical structures including optical nerves left and right and optic chiasm was 9.1 Gy in VMAT, 14.5 Gy in non-coplanar and 11.5 Gy in coplanar IMRT. Fig. 2 presents an exemplary comparative DVH comparing maximum doses to the optical system in VMAT and IMRT treatment plans of patient \#3, showing superior dose involvement of optical structures in the VMAT plan. In summa, doses to the optical system were $11.5,14.5$, and $9.1 \mathrm{~Gy}$ for coplanar, non-coplanar IMRT and VMAT.

Doses to the lenses were lowest in VMAT (5.2 Gy) and highest in non-coplanar IMRT (6.3 Gy); they were 5.9 Gy in coplanar IMRT plans.

\section{Discussion and Conclusion}

The treatment of extensive malignant scalp lesions remains a rare yet challenging and complex indication for radiotherapy. TSI, i.e. total scalp irradiation constitutes an adequate treatment for angiosarcoma, mycosis fungoides and extensive squamous cell carcinoma of the scalp $[2,6,10]$. Most commonly, conventional lateral electron-photon techniques [9,21], IMRT [10] but also tomotherapy [5], RapidArc and -in some casesHDR brachytherapy is used [22]. However, clinical presentation, the individual shape of the scalp and dose variability make it impossible to define the single best treatment for all patients and often dictate the chosen technique.

We previously reported a comprehensive dosimetric comparison of coplanar and non-coplanar photon IMRT in the treatment of extensive scalp lesions and found that noncoplanar beams increased dose homogeneity and PTV coverage and reduced doses to OARs, particularly at the optic chiasm [12].

This treatment planning study describes our institution's experience in TSI and comparatively evaluates coplanar and non-coplanar IMRT against VMAT.

Keeping treatment delivery times low, generally has the benefit of reduced intrafraction patient motion and lower patient exposure to leakage radiation. Recently, Inoue et al. [23] reported shorter TSI delivery time and reduced MU using a multi jaw-size concave arc technique. In our study which was based on a dose description of $50 \mathrm{~Gy}$ to the PTV in single fractions of 2 Gy, MUs per single fraction were lowest in VMAT (950.8) and did not differ considerably from those of non-coplanar (662.8) but were significantly lower than MUs in coplanar IMRT (974.2). In the literature, MUs for VMAT in TSI are reported ranging from 1,791 (60 Gy in single fractions of $2 \mathrm{~Gy}, 9$ arcs) to 1,188 (20 Gy in single fractions of $2 \mathrm{~Gy}, 6$ arcs) $[24,25]$.

In this work, homogeneity and target volume coverage was slightly superior in VMAT, however both PTV coverage and HI were comparable between plans. Non-coplanar IMRT plans were most conformal and dose hotspots within and outside the target volume were comparable between treatment plans despite better maximum and minimum PTV doses VMAT plans. Hu et al. [25] showed improved target volume coverage and homogeneity for both IMRT and RapidArc and Song et al. [24] reported the best conformity in tomotherapy plans $(\mathrm{Cl}$ 1.35) followed by VMAT $(\mathrm{Cl}, 1.49)$ while the photon-electron technique was least conformal $(\mathrm{Cl}, 1.73)$. Our own results indicate comparable conformity between VMAT $(\mathrm{Cl}, 1.43)$ and non-coplanar IMRT $(\mathrm{Cl}, 1.45)$, while conformity was best with the coplanar IMRT technique in our study $(\mathrm{Cl}, 1.4)$, however differences in conformity were smaller in our study compared to the data of Song et al. [24]. Analogue to the latter work, the $\mathrm{HI}$ in our study was similar across plans, yet, slightly poorer in IMRT plans compared to VMAT.

Overall, critical structures including brain, brain stem, optical system, and lenses could be spared best with VMAT which also produced the sharpest dose gradient towards healthy tissue but all critical organ doses were within clinically acceptable levels. In all patient planning cases, the optic structures received a mean dose less than 25 Gy which was also not exceeded for the brain in all except one coplanar IMRT plan. Mean doses to the lenses ranged from 4.5-8.1 Gy and never exceeded $7 \mathrm{~Gy}$ in VMAT and coplanar IMRT plans while the highest doses to the lenses were noted in a non-coplanar IMRT.

In the work of Hu et al., IMRT was inferior to 3D-conformal radiation therapy (CRT) in sparing of optical structures and RapidArc failed to improve doses to the optic system [25]. In our study, doses at the optic nerves were superior for VMAT in all patients compared to coplanar and non-coplanar IMRT while the optic chiasm could be spared best with non-coplanar IMRT. According to Hu et al. [25], doses to the lenses could be reduced by using RapidArc. VMAT in our study failed to reduce doses to the lenses compared to coplanar IMRT, however, sparing of the lenses was inferior in non-coplanar IMRT as opposed to VMAT. Song et al. [24] reported the lateral electronphoton technique to feature lower doses to optical structures compared to VMAT and helical tomotherapy however irradiated brain volume and doses to the brain were superior in VMAT which is in accordance with our results. Compared to the results of Song et al. [24], optic nerves and brain stem doses were higher in our VMAT plans while doses to the lenses were comparable. The data of $\mathrm{Hu}$ et al. [25] indicates that $3 \mathrm{D}-\mathrm{CRT}$ is clearly inferior to non-coplanar IMRT in regard of 
target coverage and brain protection where IMRT significantly deceased doses to the brain compared to 3D-CRT. In the work of Song et al. [24], brain protection was superior in VMAT and tomotherapy compared to the photon-electron approach which are further complicated by technical obstacles such as field matching.

Our own results suggest that both brain and brain stem protection was best in VMAT while the highest brain and brain stem doses were produced with the coplanar-IMRT plans.

In a clinical perspective, protection of the brain is prudent in the context of maintaining neurocognitive function in patients. In all treatment plans in our study, a mean brain dose of 25 Gy was not exceeded, however, recently there is increasing evidence that even low doses to the brain can significantly impact neurocognition [26] so that particular importance comes to protection of the hippocampus [27] which in our work could not be achieved due to the fact that treatment planning was CT-based and did not include cranial magnetic resonance imaging (MRI). MRI-based delineation, however, can help to further reduce the dose in the hippocampal region in order to prevent neurocognitive dysfunction when using VMAT for TSI. Song et al. [24] were able to show that using the lateral photon-electron or tomotherapy approach, doses to the hippocampus could be lowered compared to VMAT, however, not to the point of effective sparing of the hippocampal region.

In summa, the results of our study suggest superior target volume coverage, homogeneity, dose gradient and organ at risk protection in VMAT which provided particularly low doses to the brain and optical system. However, the variation of dosimetric parameters between patients and the small sample size in this study need to be considered in the interpretation of the results.

In the real clinical situation, additional limiting factors such as planning time, equipment availability but also the specific individual clinical presentation and both the institutes' experience and technical capacities must be considered when choosing an adequate technique for TSI.

\section{Conflict of Interest}

No potential conflict of interest relevant to this article was reported.

\section{Acknowledgments}

We would like to thank our colleagues from the Department of Radiation Oncology for their contribution to this study and their continuous support. Sources of funding for the research project came entirely from the Department of Radiation Oncology of the Martin Luther University Halle-Wittenberg.

\section{References}

1. Mendenhall WM, Mendenhall CM, Werning JW, Reith JD, Mendenhall NP. Cutaneous angiosarcoma. Am J Clin Oncol 2006;29:524-8.

2. Samant RS, Fox GW, Gerig LH, Montgomery LA, Allan DS. Total scalp radiation using image-guided IMRT for progressive cutaneous T cell lymphoma. Br J Radiol 2009;82:e122-5.

3. Wojcicka JB, Lasher DE, McAfee SS, Fortier GA. Dosimetric comparison of three different treatment techniques in extensive scalp lesion irradiation. Radiother Oncol 2009;91:255-60.

4. Tung SS, Shiu AS, Starkschall G, Morrison WH, Hogstrom KR. Dosimetric evaluation of total scalp irradiation using a lateral electron-photon technique. Int J Radiat Oncol Biol Phys 1993;27:153-60.

5. Locke J, Low DA, Grigireit T, Chao KS. Potential of tomotherapy for total scalp treatment. Int J Radiat Oncol Biol Phys 2002;52:553-9.

6. Mellenberg DE, Schoeppel SL. Total scalp treatment of mycosis fungoides: the $4 \times 4$ technique. Int J Radiat Oncol Biol Phys 1993;27:953-8.

7. Able CM, Mills MD, McNeese MD, Hogstrom KR. Evaluation of a total scalp electron irradiation technique. Int J Radiat Oncol Biol Phys 1991;21:1063-72.

8. Sagar SM, Pujara CM. Radical treatment of angiosarcoma of the scalp using megavoltage electron beam therapy. $\mathrm{Br} J$ Radiol 1992;65:421-4.

9. Akazawa C. Treatment of the scalp using photon and electron beams. Med Dosim 1989;14:129-31.

10. Bedford $J \mathrm{~L}$, Childs PJ, Hansen VN, Warrington AP, Mendes RL, Glees JP. Treatment of extensive scalp lesions with segmental intensity-modulated photon therapy. Int J Radiat Oncol Biol Phys 2005;62:1549-58.

11. Chan MF, Song Y, Burman C, Chui CS, Schupak K. The treatment of extensive scalp lesions combining electrons with intensity-modulated photons. Conf Proc IEEE Eng Med Biol Soc 2006;1:152-5.

12. Ostheimer $C_{1}$ Janich $M$, Hubsch $P$, Gerlach $R$, Vordermark D. The treatment of extensive scalp lesions using coplanar and non-coplanar photon IMRT: a single institution experience. Radiat Oncol 2014;9:82.

13. Kinard JD, Zwicker RD, Schmidt-Ullrich RK, Kaufman N, Pieters R. Short communication: Total craniofacial photon shell technique for radiotherapy of extensive angiosarcomas of the 
head. Br J Radiol 1996;69:351-5.

14. Kelly PJ, Mannarino E, Lewis JH, Baldini EH, Hacker FL. Total dural irradiation: RapidArc versus static-field IMRT: a case study. Med Dosim 2012;37:175-81.

15. Stang $K_{1}$ Alite F, Steber J, Emami B, Surucu M. Leukemia cutis of the face, scalp, and neck treated with non-coplanar split field volumetric modulated arc therapy: a case report. Cureus. 2015;7:e430.

16. Feuvret L, Noel G, Mazeron JJ, Bey P. Conformity index: a review. Int J Radiat Oncol Biol Phys 2006;64:333-42.

17. Paddick I. A simple scoring ratio to index the conformity of radiosurgical treatment plans: technical note. J Neurosurg 2000;93 Suppl 3:219-22.

18. Hodapp N. The ICRU Report 83: prescribing, recording and reporting photon-beam intensity-modulated radiation therapy (IMRT). Strahlenther Onkol 2012;188:97-9.

19. Gregoire V, Mackie TR. State of the art on dose prescription, reporting and recording in Intensity-Modulated Radiation Therapy (ICRU report No. 83). Cancer Radiother 2011;15:555-9.

20. The International Commission on Radiation Units and Measurements. J ICRU 2010;10:NP. http://doi.org/10.1093/ jicru/ndq001.

21. Hata M, Wada H, Ogino I, et al. Radiation therapy for angiosarcoma of the scalp: treatment outcomes of total scalp irradiation with $\mathrm{X}$-rays and electrons. Strahlenther Onkol 2014;190:899-904.

22. Jumeau $R$, Renard-Oldrini $S$, Courrech $F$, et al. High dose rate brachytherapy with customized applicators for malignant facial skin lesions. Cancer Radiother 2016;20:341-6.

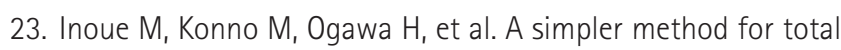
scalp irradiation: the multijaw-size concave arc technique. J Appl Clin Med Phys 2014;15:4786.

24. Song JH, Jung JY, Park HW, et al. Dosimetric comparison of three different treatment modalities for total scalp irradiation: the conventional lateral photon-electron technique, helical tomotherapy, and volumetric-modulated arc therapy. J Radiat Res 2015;56:717-26.

25. Hu J, Xiao W, He Z, Kang D, Chen A, Qi Z. Target splitting noncoplanar RapidArc radiation therapy for a diffuse sebaceous carcinoma of the scalp: a novel delivery technique. Radiat Oncol 2014;9:204.

26. Mizumatsu S, Monje ML, Morhardt DR, Rola R, Palmer TD, Fike $J R$. Extreme sensitivity of adult neurogenesis to low doses of X-irradiation. Cancer Res 2003;63:4021-7.

27. Gondi V, Tome WA, Mehta MP. Why avoid the hippocampus? A comprehensive review. Radiother Oncol 2010;97:370-6. 